\title{
6.4 MODELS OF ROTATING NEUTRON STARS IN GENERAL RELATIVITY
}

\author{
S. BONAZZOLA \\ Observatoire de Paris, 92-Meudon, France \\ and \\ G. MASCHIO \\ Instituto di Matematica Applicata, Universita di Roma, Rome, Italy
}

\begin{abstract}
We describe a numerical method of integration of Einstein's equations coupled with the hydrodynamic equations for a perfect fluid. We give, also, some preliminary results.

We point out that the analogous problem was considered by Ostriker and Mark (1968) in the Newtonian case; by Thorne (1969) in General Relativity, in the weak angular velocity approximation; by Barden and Wagoner (1969) for disk configurations in the pressure vanishing case.
\end{abstract}

\section{Introduction}

We shall describe a numerical method of integration of Einstein's equations coupled with the hydrodynamic equations for a perfect fluid. We shall give, also, some preliminary results.

We want to point out that the analogous problem was considered by Ostriker and Mark (1968) in the Newtonian case; by K. Thorne (1969) in General Relativity, in the weak angular velocity approximation; by Bardeen and Wagoner (1969) for disk configurations in the pressure vanishing case.

\section{Assumptions}

We begin by listing some properties that we should expect to characterize the geometry of the space and the perfect fluid.

(I) Space-time should have Euclidean topology.

(II) Space-time should be stationary and with axial symmetry. It should be flat at spatial infinity. Hence the space-time should admit a global 2-parameter abelian group of motion.

(III) The fluid should be considered as the source of the metric.

(IV) The fluid is assumed to be perfect so that the energy momentum tensor has the form

$$
\begin{aligned}
T^{\alpha \beta} & =(\mu+P) U^{\alpha} U^{\beta}+P g^{\alpha \beta} \\
U_{\alpha} U^{\alpha} & =1
\end{aligned}
$$

and we suppose the existence of such a state equation (in parametric form $\mu(p), P(p)$, 
$N(p))$ that the quantity

$$
N=\int_{0}^{p} \frac{\mathrm{d} P}{\mathrm{~d} p^{\prime}} \frac{\mathrm{d} p^{\prime}}{\mu\left(p^{\prime}\right)+P\left(p^{\prime}\right)}
$$

is a regular function of $p$ when $\varrho, \mu, P$ tend to zero.

\section{Properties of a Rotating Gas}

Let

$$
x^{1}=\varrho, \quad x^{2}=\phi, \quad x^{3}=z, \quad x^{0}=c t
$$

be the cylindrical coordinates adapted to the axial symmetry.

The form of the metric is

$$
\mathrm{d} s^{2}=g_{00} c^{2} \mathrm{~d} t^{2}+2 g_{02} c \mathrm{~d} t \mathrm{~d} \phi+\varrho^{2} g_{22} \mathrm{~d} \phi^{2}+g_{11} \mathrm{~d} \varrho^{2}+g_{33} \mathrm{~d} z^{2}
$$

where the $g_{\alpha \beta}$ depend only on $\varrho$ and $z$.

We have from the motion equations of the fluid:

$$
\nabla_{\alpha} T_{\beta}^{\alpha}=0 \Leftrightarrow U^{\alpha} \nabla_{\alpha} U_{\beta}+\partial_{\beta} N=0
$$

so that the vector $U^{\alpha} \nabla_{\alpha} U_{\beta}$ has to be a free curl vector.

We shall try to satisfy the Equation (1) by choosing the vector $U^{\alpha}$ in the following form

$$
\begin{aligned}
U^{\alpha} & =(0, \Omega, 0,1) / \Gamma \\
& =\left(g_{00}+2 g_{02} \Omega+\Omega^{2} g_{22}\right)
\end{aligned}
$$

where $\Gamma^{2}$ is obtained by the condition $U_{\alpha} U^{\alpha}=-1$.

From the Equation (1) we obtain

$$
\frac{1}{2} \frac{\Omega^{2} \partial_{\alpha} g_{22}+2 \Omega \partial_{\alpha} g_{02}+\partial_{\alpha} g_{00}}{\Omega^{2} g_{22}+2 g_{02} \Omega+g_{00}}+\partial_{\alpha} N=0 .
$$

We can satisfy the equation (2) in the following cases:

\section{A. RIGID MOTION}

This case is characterized by $\Omega=$ constant. We have the General Relativistic Bernouilli's theorem (Boyer, 1965; Lichnerowicz, 1955).

$$
N+\frac{1}{2} \log \left(g_{00}+2 \Omega g_{02}+\Omega^{2} g_{22}\right)+K=0
$$

where $K$ is an integration constant.

As an important consequence of this theorem, we can obtain without any integration, the energy momentum tensor as a function of two parameters, $\Omega$ and $\mu(0,0)$ knowing the $g_{\alpha \beta}(K$ can be related to the density of the fluid at the center, $\mu(0,0))$. 


\section{B. DIFFERENTIAL MOTION}

From the Equation (2) we have

If we put

$$
\partial_{\alpha}\left(N+\frac{1}{2} \log \left(\Omega^{2} g_{22}+2 \Omega g_{02}+g_{00}\right)\right)-\frac{\left(\Omega g_{22}+g_{02}\right) \partial_{\alpha} \Omega}{\Omega^{2} g_{22}+2 \Omega g_{02}+g_{00}}=0 \text {. }
$$

$$
\frac{\Omega g_{22}+g_{02}}{\Omega^{2} g_{22}+2 \Omega g_{02}+g_{00}}=f(\Omega) / \Omega
$$

where $f(\Omega)$ is an arbitrary function of $\Omega$, we obtain the General Relativistic Bernouilli's theorem for a fluid in differential rotation

$$
0=\partial_{\alpha}\left(N+\frac{1}{2} \log \left(\Omega^{2} g_{22}+2 \Omega g_{02}+g_{00}\right)+\int \frac{f(\Omega) \mathrm{d} \Omega}{\Omega}\right)
$$

The solution of the Equation (3) gives us

$$
\Omega=\Omega\left(g_{\alpha \beta}(\varrho, z)\right) .
$$

Let us give two examples of differential motion

(I) $f(\Omega)=\Omega_{0}=$ constant

We have

$$
\Omega=\frac{\left(2-\Omega_{0}\right) g_{02} \mp \sqrt{g_{02}^{2}\left(\Omega_{0}-2\right)^{2}+4 g_{00} g_{22}\left(\Omega_{0}-1\right)} \Omega_{0}}{2 g_{22}\left(\Omega_{0}-1\right)} .
$$

By taking in account that, when $\varrho$ tends to zero, $g_{02}$ and $g_{22}$ tend to zero as $\varrho^{2}$, we have

$$
\lim _{\varrho \rightarrow 0} \Omega \propto \frac{1}{\varrho}
$$

i.e. a vortex.

(II) Except in a few simple cases, it is not easy to solve the Equation (3). For this reason, we consider a system composed of shells of fluid in rigid rotation. The shells have different angular velocities.

For that, we have to solve the following problem: find in a fluid in rigid motion, the two surfaces $S$ across which the angular velocity $\Omega$ can jump. To have a stationary motion, it is easy to show that the pressure $P$ of the fluid must be continuous across $S$. Let $\Delta$ be the jump of $\Omega$ across $S$. Let us call $V_{1}$ the region in which the angular velocity is equal to $\Omega$ and $V_{2}$ the region in which the angular velocity is equal to $\Omega+\Delta$.

From the equation $P_{1}(S)=P_{2}(S)$ we obtain the equation of the surface $S$

$$
\frac{g_{22}\left(\Delta^{2}+2 \Omega_{1} \Delta\right)+2 g_{20} \Delta}{g_{00}+g_{22} \Omega^{2}+2 g_{20} \Omega}=\text { const . }
$$

Of course, in the Newtonian limit, we have the Poincaré's theorem: the surfaces across which the angular velocity can change are cylinders. This result is quite useful for numerical applications. 


\section{Integral form of Einstein's Equations}

The Einstein's equations can be written in integral form

$$
\begin{aligned}
\xi & =-\frac{1}{4 \pi} \int G_{1}\left(x, x^{\prime}\right)\left[\chi A^{2}\left(T_{\overline{0} \overline{0}}-\frac{1}{2} T_{\alpha}^{\alpha}\right)-Q_{00}\right] \varrho^{\prime} \mathrm{d} \varrho^{\prime} \mathrm{d} z^{\prime} \mathrm{d} \phi^{\prime} \\
\Phi & =-\frac{1}{4 \pi \varrho} \int G_{3}\left(x, x^{\prime}\right)\left[\frac{2 \chi A^{2}}{B \varrho^{2}} T_{\overline{0} \overline{2}}+Q_{02}\right] \varrho^{\prime 2} \mathrm{~d} \varrho^{\prime} \mathrm{d} z^{\prime} \mathrm{d} \phi^{\prime} \\
C B & =1+\frac{1}{2 \pi \varrho} \int \chi\left(T_{1}^{1}+T_{3}^{3}\right) A^{2} B C \varrho^{\prime} G_{2}\left(x, x^{\prime}\right) \mathrm{d} \varrho^{\prime} \mathrm{d} z^{\prime} \\
\log (A B) & =\frac{1}{4 \pi} \int\left[A^{2} \chi\left(T_{2}^{2}+T_{\overline{2}}^{2}+T_{0}^{0}-T_{\overline{0}}^{\overline{0}}\right)+Q_{11}\right] G_{0}\left(x x^{\prime}\right) \mathrm{d} \varrho^{\prime} \mathrm{d} z
\end{aligned}
$$

where

$$
\begin{aligned}
& \xi=\frac{1}{2} \log \left(g_{00}\right), \quad \Phi=g_{02} /\left(g_{00} \varrho^{2}\right) \\
& B=\sqrt{g_{00}} ; \quad A=\sqrt{-g_{11}}, \quad C=\frac{1}{\varrho} \sqrt{-g_{22}+\left(g_{02}\right)^{2} / g_{00}}
\end{aligned}
$$

$G n\left(x ; x^{\prime}\right)$ are the Green's functions of the elliptic operators

$$
\partial^{2} \varrho+\partial^{2} z+\frac{n}{\varrho} \frac{\partial}{\partial \varrho} \quad(n=0,1,2,3)
$$

Let $\gamma_{\alpha}, \gamma_{\alpha \beta}$ be

$$
\gamma_{\alpha}=g_{\alpha} / \sqrt{ } g_{00} ; \quad \gamma_{\alpha \beta}=g_{\alpha \beta}-\gamma_{\alpha} \gamma_{\beta}
$$

The quantities $T_{00}, T_{0 \alpha}$, and $T_{\alpha \beta}$ are defined by the relations:

$$
\begin{aligned}
& T_{00}=\gamma_{\alpha} \gamma_{\beta} T^{\alpha \beta} ; \quad T_{\beta 0}=\gamma_{\alpha} \gamma_{\delta \beta} T^{\alpha \delta} \\
& T_{\alpha \beta}=T^{\gamma \delta} \gamma_{\gamma \alpha} \gamma_{\beta \delta}
\end{aligned}
$$

The terms $Q_{\alpha \beta}$ are the quadratic terms of the derivatives of $g_{\alpha \beta}$.

We can integrate the System (5) in a selfconsistent way: we first obtain the functions $\xi, \Phi, A, C$ by using a trial distribution of matter ( $g_{\alpha \beta}$ being the flat metric); then by using the hydrodynamical Equation (2a) or (2b) we compute a new distribution of matter, we get the terms $Q_{\alpha \beta}$ by using the quantities $\xi, \Phi, A, C$; we compute again the functions $\xi, \Phi, A, C$, the new distribution of matter and so on.

We do not discuss here the convergence of the method.

We have got ready a computer program in order to obtain solutions of the Einstein's equations.

The calculations were performed on an Univac 1108 Computer. The method converges very well, and the time spent to get one solution is about five minutes. The number of the cycles is about four. The precision is 4 figures for $g_{00}, g_{11}, g_{22}$ and 3 figures for $g_{02}$.

The precision decreases a little when the eccentricity of the fluid increases. 
Until now, only a few solutions were obtained. We can give some results in the case of rigid motion and for a free Fermi gas equation of state: for a star of 0.6 solar mass the maximum of the eccentricity (the ratio between maximum radius and minimum radius) is 1.5 , and the period $3 \mathrm{msec}$ (only 10 times less then the period of the Crab pulsar). The ratio $K=Q M / J^{2}$ is $\simeq 10^{4}$ ( $Q$ is the quadrupole momentum, $M$ is the mass of the star, $J$ is the angular momentum).

We remember that $K$ is equal to 1 in the Kerr's metric. Because of the large value of $K$ in our solution, it seems that a perfect fluid in rotation cannot be a source of the Kerr's metric. Much work has still to be done: we need more solutions to get relationships of the kind: $N\left(\mu_{0}, \Omega\right), M\left(\mu_{0}, \Omega\right), W\left(\mu_{0}, \Omega\right), J\left(\mu_{0}, \Omega\right)$ where $N, M, W, J, \mu_{0}, \Omega$, are respectively the barionic number of the star, the global mass, the global kinetic energy, the global angular momentum. All these global quantities must be obtained as functions of the central density $n_{0}$ and the angular velocity $\Omega$.

Another relationship would be quite interesting: the critical mass of the star as a function of the angular velocity, for rigid motion, as for differential motion.

\section{Applications to Different Problems}

The solutions obtained for the equilibrium configurations make possible the approach to a new class of problems.

(a) Stability of a rotating object in general relativity, by using the equilibrium solution as a background.

(b) Problem of gravitational collapse: we remember that for the problem of the evolution of the Einstein's equations, the Cauchy's data cannot be given arbitrarily: they have to be a solution of the equation

$$
R^{0 i}=\chi T^{0 i}
$$

We can show that the $g_{\alpha \beta}$ for the equilibrium solutions are good Cauchy-data for the collapse problem, relative to a very well defined physical situation. This is the first step to solve the more difficult problem of the collapse.

\section{References}

Bardeen, J. M. and Wagoner, R. V.: 1969, Astrophys. J. 158, L65.

Boyer, R. H.: 1965, Proc. Cambridge Phil. Soc. 61, 527.

Lichnerowicz, A.: 1955, Théories de la gravitation et de l'electromagnétisme, Masson, Paris.

Ostriker, J. P. and Mark, J. W. K.: 1968, Astrophys. J. 151, 1075.

Thorne, K.: 1969, Astrophys. J. 153, 807.

\section{Discussion}

A. G. W. Cameron (to Dr. Chanmugam): The vibrational periods calculated for a model which are imaginary using a composition-changing equation of state can certainly be found real for a compositionpreserving equation of state. This simply means that a model can vibrate in a condition in which it is secularly unstable and is on the way to collapse.

G. Chanmugam: The question of long term effects and secular stability is as in the case of ordinary stars is a very difficult one as non-linear effects are important and needs detailed separate discussion. 
D. W. Richards: Can the various theorists come to any agreement on the upper and lower limits of the mass and radius for the star NP 0532? If so, what are these limits?

Unanimous reply: No.

J.P. Ostriker (to Dr. Cohen): If I remember an earlier version of this work correctly, you found a different moment of inertia appropriate for angular momentum $(J)$ and kinetic energy $(T)$. That result seems inconsistent with the relation $T=\frac{1}{2} \omega J$ which you gave today (and which is I believe right). Has your opinion changed?

J. M. Cohen: These results are the same. My expressions for the angular momentum and rotational energy are the same as those in J. Math. Phys. 8 (1967) 1477, and those in Astrophys. Space Sci.

$V$. Canuto (to Dr. Chanmugam): Did you compute the value of the nuclear compressibility? It seems to me that it is more sensitive to compare second derivatives of the energy than first, since they all look alike and yet they give quite different stable neutron star masses.

G. Chanmugam: We use Nemeth and Sprung's results. They do match the binding energy with nuclear matter but I don't know whether they consider the compressibility. 